\title{
Thiol Disulfide Homeostasis of Pediatric Oncology Patients After the Positron Emission Tomography/ Computerized Tomography Imaging: A Cross-Sectional Study
}

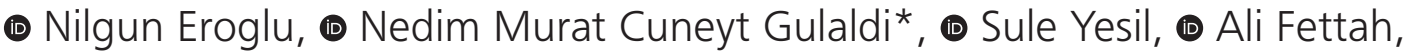 \\ (1) Burcak Bilgin Kurucu, (1) Emre Capkinoglu, (1) Ozcan Erel** , (1) Gurses Sahin \\ Dr. Sami Ulus Maternity and Children Training and Research Hospital, Clinic of Pediatric Hematology and Oncology, Ankara, Turkey \\ *Ministry of Health, Ankara City Hospital, Clinic of Nuclear Medicine, Ankara, Turkey \\ **Yildirim Beyazit University, Department of Medical Biochemistry, Ankara, Turkey
}

Abstract

\begin{abstract}
Aim: Positron emission tomography/computerized tomography (PET/CT) is used for oncologic imaging as hybrid imaging. Pediatric patients are exposed to ionizing radiation when they undergo hybrid medical imaging examinations. It is especially important to minimize the radiation exposure when children and infants are imagined, as immature tissues are more susceptible to damage induced by radiation. This study aims to reveal the oxidant and antioxidant status in oncology patients before and after PET/CT imaging.

Methods: Between February 2019 and July 2019, pediatric oncology patients who underwent PET imaging at the time of diagnosis in our center were included in the study. Eighteen newly diagnosed cancer pediatric patients participated in this prospective crosssectional study. Data were saved to synchronously kept excel log files and used in the study. Disulphide amounts, total and native thiols, native thiol/total thiol percent ratios, disulphide/native thiol percent ratios, disulphide/total thiol percent ratios, and Ischemia-Modified Albumin were calculated before and after the PET/CT imaging
\end{abstract}

Results: Disulphide levels, disulphide/total thiol and disulphide/native thiol ratios of patients' serum samples were significantly higher and the ratio of native thiol/total thiol was reduced after the PET/CT.

Conclusion: There was disturbed thiol-disulfide homeostasis and the balance changed in the direction of oxidant damage. Our results indicate the possible oxidative stress condition due to radiation exposure with PET/CT in pediatric oncology patients.

Keywords: Thiol disulfide homeostasis, ionizing radiation, PET/CT

\section{Introduction}

Positron emission tomography (PET) is a noninvasive imaging procedure and it is useful for clinical diagnosis, staging and treatment (1). According to the recent reports, there are significantly higher specificity, sensitivity, and accuracy in the hybrid PET/computerized tomography (CT) imaging than those in the conventional imaging for evaluation of the pediatric malignancies $(2,3)$. A baseline PET/CT scan is also the best tool available for subsequent assessment of response. PET/CT enables the characterization and detection of disease in the early stages and gives worthy information for diagnosis not easily obtained based on the traditional techniques for imaging (4-6).

Stochastic effects such as cancer are due to translocations in cells and radiation-induced DNA mutation. These effects follow a hidden period between 2 and 30 years (7). Due to longer life periods of children for the deleterious stochastic effects after irradiation, they are more exposed toin coordin ionizing radiation than adults. Therefore, radiation exposure during PET/

Address for Correspondence: Nilgun Eroglu, Dr. Sami Ulus Maternity and Children Training and Research Hospital, Clinic of Pediatric Hematology and Oncology, Ankara, Turkey Phone: +90 3123176182 E-mail: dr-nilguner@hotmail.com ORCID: orcid.org/0000-0002-9956-4054 Received: 15.04.2021 Accepted: 23.05.2021 
CT imaging is important in pediatric patients in terms of oxidant damage. Although the oxidant damage of ionizing radiation is known, the effect after PET/CT imaging is not fully known.

The antioxidant defense system inhibits the oxidative effects of free radicals. There is a sulfhydryl group in thiols with an essential role in the coordination of the antioxidant defense system. Oxidation reaction can occur in the thiols throughout oxidant, forming disulphide bonds. When there is oxidative stress, sulfhydryl groups are changed into disulphide bridges subsequently converted into thiols again. There is a balanced continuation of the cycle. Thus, thiol disulfide homeostasis (TDH) is kept. TDH is essential in apoptosis, oxidative stress, protection against antioxidants, detoxification, enzymatic activity, and cellular signal transmission (8).

In the present study, thiol levels were evaluated in cancer patients to show oxidant-antioxidant balance due to radiation exposure before and after PET/CT imaging. To our knowledge this is the first study, presenting data that cancer patients oxidative stress status with thiol/disulfide homeostasis.

\section{Methods}

\section{Study Design}

Approval from the University of Health Sciences, Ankara Child Health and Diseases Hematology Oncology Training and Research Hospital Ethics Review Committee obtained (no: 2019-019). Between February 2019 and July 2019, pediatric oncology patients who underwent PET imaging at the time of diagnosis in Ankara Child Health and Diseases Hematology Oncology Training and Research Hospital were included in the study. Eighteen newly diagnosed cancer pediatric patients participated in this prospective study. Data were saved to synchronously kept excel log files and used in the study. Written informed consent was obtained from all patients before participation in the study.

Inclusion criteria were: newly diagnosed cancers; NonHodgkin lymphoma, Hodgkin lymphoma, neuroblastoma, Langerhans cell histiocytosis, primitive neuro-ectodermal tumor, rhabdomyosarcoma, Wilms tumor and exclusion criteria were: scans required or recommended at primary site such as magnetic resonance imaging (MRI), Ultrasound, ECHO, CT other soft tissue sarcomas, scans for patients who relapsed. Patients with clinical findings such as infection, fever and vomiting that may cause oxidant damage were excluded from the study too.

Each patient was required to be admitted as at least 6 hours of fasting. Blood glucose levels were measured in each child before radiopharmaceutical injection. Two hundred $\mathrm{mg} / \mathrm{dL}$ of blood glucose level was used as criteria for postponing the imaging procedure. F-18
Fluorodeoxyglucose (F-18 FDG) calculated as 3-5 MBq/ $\mathrm{kg}$ body weight was injected intravenously to the child. They were advised to rest i low light and quiet room for approximately 45-60 min after injection. CT, PET and PET/ $\mathrm{CT}$ fusion images were taken through vertex to mid-thigh scanning on 3D PET/CT device (Philips Gemini TF 16 w/TOF Performance PET/CT, Philips Medical Systems Nederland B.V.). Images were interpreted by a nuclear medicine physician experienced in pediatric studies. For the staging of newly diagnosed patients, $2 \mathrm{~mL}$ of blood was taken from the patients half an hour before and 2 hours after PET imaging and thiol metabolite measurements were made.

\section{Measurement of Thiol/Disulphide Homeostasis}

An automated spectrophotometric method which was described by Erel and Neselioglu (9) was used to measure the Thiol/Disulphide Homeostasis tests. For short, free functional thiol groups were formed with sodium borohydride by reducing the disulphide bonds. The reduction of 5.5'-dithiobis-(2-nitrobenzoic) acid (DTNB) was prevented by consuming and removing the reductant sodium borohydride which has not been used with formaldehyde, and all of the thiol groups, including native and reduced thiol groups, were specified after reacting with DTNB. The dynamic disulphide amount is provided by half of the difference between the native thiols and total thiols. After the native and total thiols were specified, disulphide/native thiol percent ratios (SS/SH), disulphide/ total thiol percent ratios (SS/SH+SS), disulphide amounts, and native thiol/total thiol percent ratios $(\mathrm{SH} / \mathrm{SH}+\mathrm{SS})$ were measured (9).

\section{Ischemia-Modified Albumin Measurement}

Venous blood samples were used during admittance within one hour to obtain the measurement of IschemiaModified Albumin (IMA) levels. Specimens were kept at room temperature for 30 minutes and then centrifuged for five minutes at 3,500 rpm. Later samples were taken to Eppendorf tubes and kept at $-80{ }^{\circ} \mathrm{C}$ for analysis. The Albumin Cobalt Binding test was performed to detect the presence of IMA. Fifty $\mathrm{mL} 0.1 \%$ cobalt (II) chloride $\left(\mathrm{CoCl}_{2}, 6 \mathrm{H}_{2} \mathrm{O}\right)$ (Steinheim, Sigma-Aldrich Chemie $\mathrm{GmbH}$ Riedstrasse 2, Germany) was added to the patient serum to perform this test. After mixing, $50 \mathrm{~mL} 1.5 \mathrm{mg} / \mathrm{mL}$ dithiothreitol was added before 10 minutes of incubation for albumin cobalt binding. After mixing, the binding capacity was reduced by adding $1.0 \mathrm{~mL}$ of a $0.9 \%$ sodium chloride solution before incubation for two minutes. Instead of dithiothreitol, the blank was prepared like the distilled water. A spectrophotometer was used to measure the sample's absorbance at $470 \mathrm{~nm}$. The results were given as absorbance units (ABSU) (10). 


\section{Statistical Analysis}

SPSS 20.0 package program was used for statistical analysis was done with. The frequencies and percentages are given in the evaluation of qualitative data. During the analysis of quantitative data, the Shapiro-Wilk test was applied and as a result of this analysis, it was observed that some parameters were normally distributed and some parameters were not normally distributed.

For data distributed normally, paired samples test was used and for data not normally distributed, The Wilcoxon-Signed rank test was used. Receiver operating characteristics (ROC) analysis was performed for the parameters that were found to be significant in these two tests. All statistical calculations were evaluated at a significance level of $p<0.05$ at a $95 \%$ confidence interval.

\section{Results}

Oncology patients participating in the study, $44.4 \%$ (8) were girls and $55.6 \%$ (10) were boys. The children's average age in this study was 11.3 years from 6.0 to 16 years. Patients diagnosis were; non-Hodgkin lymphoma $(n=6,33.3 \%)$, hodgkin lymphoma $(n=5,27.8 \%)$, neuroblastoma $(n=2,11.1 \%)$, Langerhans cell histiocytosis $(n=2,11.1 \%)$, primitive neuro-ectodermal tumor $(n=1$, $5.6 \%)$, rhabdomyosarcoma $(n=1,5.6 \%)$, Wilms tumor $(n=1,5.6 \%)$.

The radiation dose exposed by $\mathrm{CT}$ imaging in PET/ CT was $6.37 \mathrm{mSV}$, the radiation dose exposed after PET imaging was $2.53 \mathrm{mSV}$, and the total radiation dose was $8.9 \mathrm{mSv}$.

No statistically significant difference was found in Total thiol $(\mu \mathrm{mol} / \mathrm{L})$, Native thiol $(\mu \mathrm{mol} / \mathrm{L}), \mathrm{IMA}(\mathrm{ABSU})$ and albumin ( $g r / d L)$ values before and after PET/CT ( $p>0.05)$.

Before PET/CT disulfide level $(\mu \mathrm{mol} / \mathrm{L})$ was median (min-max): 8.12 (1.25-13.30), after PET/CT disulfide level $(\mu \mathrm{mol} / \mathrm{L})$ was median (min-max): 14.15 (1.35-23.55) (Table 1).

Disulfide-native thiol ratio, disulfide level, and disulfidetotal thiol ratio after PET/CT were found to be higher than before PET/CT ( $p=0.001, p=0.002, p=0.002)$ (Figure $1,2,3)$. Native thiol/ total thiol ratio before $P E T / C T$ was higher than after PET/CT $(p=0.02)$.

When the cut-off value was accepted as 93.45 caused by the native thiol/ total thiol values of the patients in the study, the sensitivity was calculated as $72.20 \%$ and specificity $33.30 \%$. The ROC curve of the disulfide and thiol values is given in Figure 4.

\section{Discussion}

In the present study, the aim was to evaluate homeostasis of thiol disulfide in pediatric oncology patients who underwent PET/CT imaging in a pediatric hematology oncology clinic before and after extraction. When free oxygen radicals are excessively produced, there will be

\begin{tabular}{|c|c|c|c|}
\hline & $\begin{array}{l}\text { Before PET/CT } \\
(n=18)\end{array}$ & After PET/CT $(n=18)$ & \\
\hline & Median (min, max) & Median (min, max) & \\
\hline $\begin{array}{l}\text { Native } \\
\text { thiol } \\
\text { ( } \mu \mathrm{mol} / \mathrm{L})\end{array}$ & $332.20(152.6-407.5)$ & $287.70(148.20-433.50)$ & 0.824 \\
\hline $\begin{array}{l}\text { Disulfide } \\
\text { ( } \mu \mathrm{mol} / \mathrm{L})\end{array}$ & $8.12(1.25-13.30)$ & $14.15(1.35-23.55)$ & 0.001 \\
\hline $\begin{array}{l}\text { IMA } \\
\text { (ABSU) }\end{array}$ & $0.62(0.38-1.08)$ & $0.62(0.34-1.25)$ & 0.691 \\
\hline
\end{tabular}

IMA: Ischemia-Modified Albumin, Paired Sample's t-test has been applied: Disulfide level after PET/CT was found to be higher than before PET/CT. PET/CT: Positron emission tomography/computerized tomography, min, max: Minimum, maximum, ABSU: Absorbance units

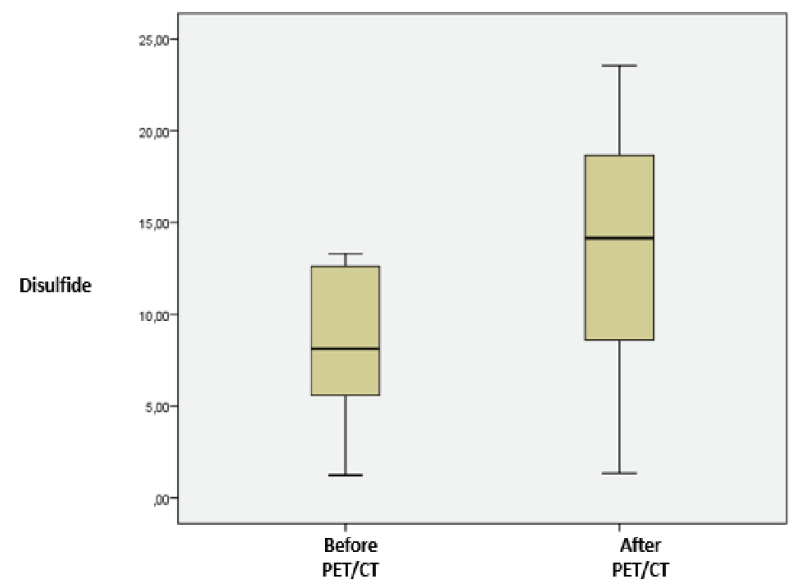

Figure 1. Distribution of disulfide values before and after PET/CT $\mathrm{PET} / \mathrm{CT}$ : Positron emission tomography/computerized tomography

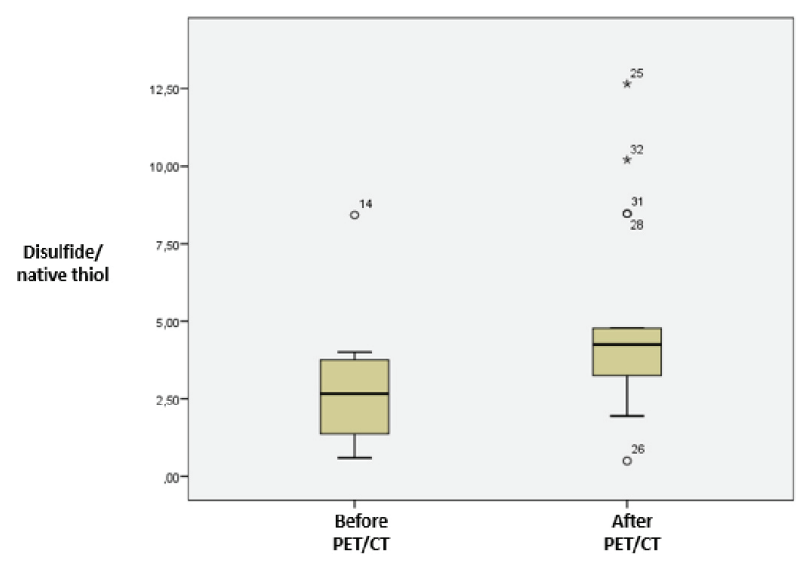

Figure 2. Distribution of disulfite/ nativethiol values before and after $\mathrm{PET} / \mathrm{CT}$

$\mathrm{PET} / \mathrm{CT}$ : Positron emission tomography/computerized tomography 


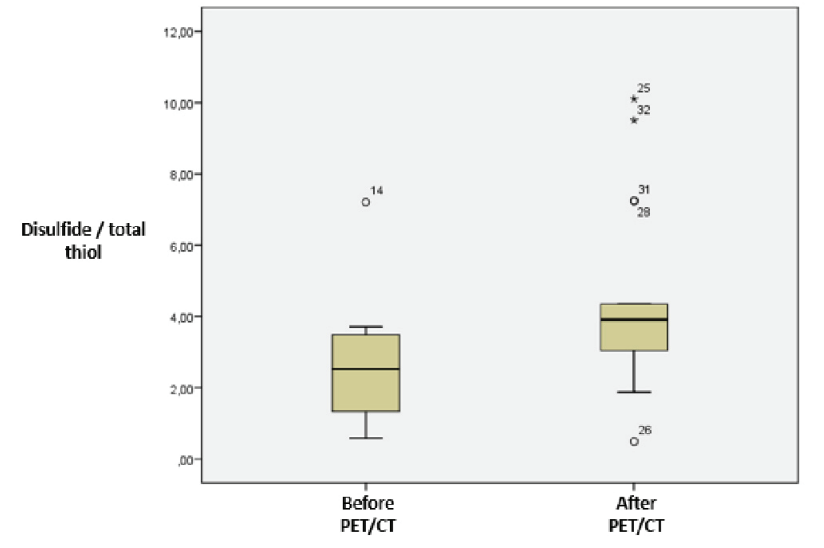

Figure 3. Distribution of disulfide /total thiol values before and after PET/CT

$\mathrm{PET} / \mathrm{CT}$ : Positron emission tomography/computerized tomography

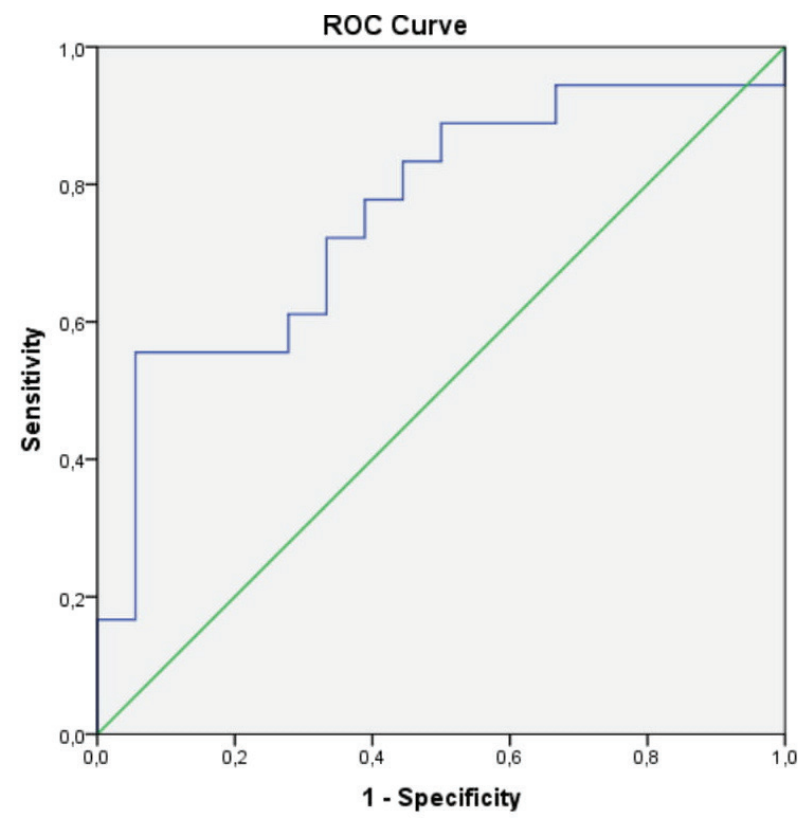

Figure 4. Native thiol/ total thiol values ROC curve ROC: Receiver operating characteristics

PET/CT: Positron emission tomography/computerized tomography

systemic damage after exposure. It causes changes the prooxidant/antioxidant balance of the tissues, ultimately causes the oxidation of the basic structures of the cell. It is important to evaluate TDH to radiation exposure on $\mathrm{PET} / \mathrm{CT}$. According to our results, imaging methods that may have lower radiation exposure such as PET/MR may be preferred in pediatric patients. As far as we know, this study is the first one in the literature.

$\mathrm{PET} / \mathrm{CT}$ studies show considerable exposure to radiation, particularly for the children who undergo followup exams regularly $(11,12)$. There is a significant variation radiation dose depending on the number of the performed PET/CT studies (13). According to the results, the CT part of the examination causes the largest radiation dose. The pediatric malignancies treatment has different and considerable long-term effects. These include avascular necrosis of the hip, the development of cardiomyopathy, early onset of heart disease, cognitive delay, higher risk of secondary malignancies, and pulmonary fibrosis. Children receiving radiation and alkylating chemotherapeutic agents as their treatment regimens are exposed to the development of secondary malignancies. The significant issue of exposure to radiation in children increases with enhancing use of PET/CT in managing the pediatric malignancies, particularly when children are reported to have a higher risk of developing secondary malignancies caused by exposure to radiation compared with adults $(14,15)$. In the present study, the radiation dose exposure by $\mathrm{CT}$ imaging in PET/CT was $6.37 \mathrm{mSV}$, the radiation dose exposed after PET imaging was $2.53 \mathrm{mSV}$, and the total radiation dose was $8.9 \mathrm{mSv}$. Therefore, to evaluate whether the radiation dose exposed in patients changes the oxidant/antioxidant balance, we evaluated oxidant/ antioxidant status of pediatric new diagnostic oncology patients with the measurement of the thiol levels before and after the PET/CT imaging.

Mercaptans or thiols are a group of organic compounds containing a sulfhydryl group containing hydrogen and sulfur atoms bound to a carbon atom. There are albumin thiols in the plasma pool, and other protein thiols contributed by thiols with low molecular weight such as cysteine, cysteinyl glycine, glutathione, homocysteine, and $\gamma$-glutamylcysteine (8).

Cellular and tissue injury due to reactive oxygen species is prevented through the reaction between organic thiol compounds and free radicals. When there is oxidative stress, sulfhydryl groups are changed into disulphide bridges subsequently converted into thiols again. There is a balanced continuation of the cycle. Thus, TDH is kept. TDH is essential in apoptosis, oxidative stress, protection against antioxidants, detoxification, enzymatic activity, and cellular signal transmission (8). This study found no statistically significant difference before and after the PET/ $\mathrm{CT}$ imaging in native and total thiol values but disulphide levels, disulphide/native thiol, and disulphide/total thiol ratios increased significantly after the $\mathrm{PET} / \mathrm{CT}$ imaging $(p=0.001, p=0.002, p=0.002)$. Native thiol/total thiol ratio was reduced after the PET/CT imaging $(p=0.02)$ and native thiol/total thiol values of the patients in the study, the sensitivity was calculated as $72.20 \%$ and specificity $33.30 \%$ with ROC curve. The reason is the high levels of oxidant radicals in patients exposed radiation after the PET/ $C T$ imaging. Arıcan et al. (16) were investigated the effect of low dose ionizing radiation exposure on thiol/disulfide homeostasis and ischemia modified albumin levels. 
They were found no statistically significant difference in terms of IMA levels in radiation exposed group like the present study.

Children are widely known to be more exposed to the radiation effects than adults. Not only are children's soft tissues and organs have more radiosensitivity than the adults since the cell is divided rapidly, but children have a longer life expectancy after exposure to show adverse effects of radiation after radiation exposure. It is hardly surprising that the risk of causing fatal cancer per exposure unit at 10 years is estimated to be about 5-15 times as much as that at 70 years, and this risk is seven times as much as that for younger children (17).

In some studies, there is an increase in the activity of antioxidant enzymes such as catalase (CAT), glutathione peroxidase (GPx), superoxide dismutase, and GPx to be protected against the higher free radicals. In other studies, long-term exposure to chronic oxidative stress and low ionizing radiation is shown to reduce antioxidant defense among workers $(18,19)$. As far as we know, disulfide level, disulfide/total thiol and native thiol/total thiol, and disulfide/native thiol ratios in pediatric oncology patients were first determined before and after the PET/ CT imaging. It was found that in this study, thiol-disulfide homeostasis was disturbed and the balance changed in the direction of oxidant damage, in radiation exposure.

The importance of increasing exposure to radiation as shown in the serial PET/CT scans for children receiving high-dose radiation therapy as part of their treatment is not clear. Whole-body PET/CT remains as an important non-invasive staging/diagnostic modality for malignancies (20). PET/MRI scanners can be used to reduce the exposure to radiation from $\mathrm{PET} / \mathrm{CT}$ imaging studies in cancerous children (21).

The diagnostic performance of F-18 FDG PET/MRI was compared with that of F-18 FDG PET/CT by Sher et al. (22) in a cohort of pediatric patients suffering from lymphoma. They showed that the two modalities had no statistical difference in lesion detection. Classification accuracy of lymph node groups was not different as negative or positive for lymphoma to us involvement. According to our results, PET/CT imaging in pediatric patients with cancer can cause oxidative stress. Considering other studies in the literature, PET/MR may be preferred.

\section{Study Limitations}

Our study has some limitation; sample groups were small. Oxidative stress could not be evaluated with different parameters such as reduced glutathione content, glutathione S-transferase activity, and malondialdehyde levels and not compared with thiol groups. The technical variations were limited using the PET scanner technology for PET/MR examination. Therefore, we could not compare the thiol groups between PET/CT and PET/MR. Despite these limitations, the study will contribute to the literature with oxidative damage by evaluating the thiol groups before and after PET/CT imaging.

\section{Conclusion}

The exposure to radiation from studies of serial PET/CT in pediatric malignancies may be regarded as significant. Our results indicate the possible oxidative stress condition due to radiation exposure with $\mathrm{PET} / \mathrm{CT}$ in pediatric oncology patients. PET/CT remains an important modality of surveillance, noninvasive diagnostic, and staging for specific pediatric malignancies. The decision should be made to use PET/CT in children individually who particularly know the exposure to cumulative radiation and the total benefit of the scan. It is concluded that pediatric patients with cancer can be evaluated using the PET/MR due to its equivalent information at a considerable radiation dose savings compared with PET/CT.

\section{Authorship Contributions}

Concept: N.E., Design: N.E., G.S., N.M.C.G., Data Collection or Processing: S.Y., A.F., B.B.K., E.C., Analysis or Interpretation: O.E., Literature Search: N.E., Writing: N.E.

Conflict of Interest: No conflict of interest was declared by the authors.

Financial Disclosure: The authors declared that this study received no financial support.

\section{References}

1. Gambhir SS. Molecular imaging of cancer with positron emission tomography. Nat Rev Cancer 2002;2:683-93.

2. Barrington SF, Mikhaeel NG, Kostakoglu $L$, et al. Role of imaging in the staging and response assessment of lymphoma: consensus of the International Conference on Malignant Lymphomas Imaging Working Group. J Clin Oncol 2014;32:3048-58.

3. Cheson $\mathrm{BD}$, Fisher RI, Barrington $\mathrm{SF}$, et al. Recommendations for initial evaluation, staging, and response assessment of Hodgkin and non-Hodgkin lymphoma: the Lugano classification. J Clin Oncol 2014;32:3059-68.

4. Tatsumi M, Miller JH, Wahl RL. 18F-FDG PET/CT in evaluating non-CNS pediatric malignancies. J Nucl Med 2007;48:192331.

5. Treves ST, Baker A, Fahey FH, et al. Nuclear medicine in the first year of life. J Nucl Med 2011;52:905-25.

6. Kleis M, Daldrup-Link H, Matthay K, et al. Diagnostic value of $\mathrm{PET} / \mathrm{CT}$ for the staging and restaging of pediatric tumors. Eur J Nucl Med Mol Imaging 2009;36:23-36.

7. Mothersill C, Seymour C. Implications for human and environmental health of low doses of ionising radiation. J Environ Radioact 2014;133:5-9. 
8. Eroglu H, Turgal M, Senat A, Karakoc G, Neselioglu S, Yucel A. Maternal and fetal thiol/disulfide homeostasis in fetal growth restriction. J Matern Fetal Neonatal Med 2021;34:1658-65.

9. Erel O, Neselioglu S. A novel and automated assay for thiol/ disulphide homeostasis. Clin Biochem 2014;47:326-32.

10. Bar-Or D, Lau E, Winkler JV. A novel assay for cobalt-albumin binding and its potential as a marker for myocardial ischemia-a preliminary report. J Emerg Med 2000;19:311-5.

11. Prasad KN, Cole WC, Hasse GM. Health risks of low dose ionizing radiation in humans: a review. Exp Biol Med (Maywood) 2004;229:378-82.

12. Steinert $M$, Weiss $M$, Gottlöber $P$, et al. Delayed effects of accidental cutaneous radiation exposure: fifteen years of follow-up after the Chernobyl accident. J Am Acad Dermatol 2003;49:417-23.

13. Alkhorayef M. Effective radiation doses in pediatric PET/CT examinations: Pilot study. Appl Radiat Isot 2021;168:109412.

14. Chawla SC, Federman N, Zhang D, et al. Estimated cumulative radiation dose from $\mathrm{PET} / \mathrm{CT}$ in children with malignancies: a 5-year retrospective review. Pediatr Radiol 2010;40:681-6.

15. Picardi M, Cavaliere C, Della Pepa R, et al. PET/MRI for staging patients with Hodgkin lymphoma: equivalent results with PET/CT in a prospective trial. Ann Hematol 2021;100:152535.

16. Arıcan S, Dertli R, Baktik S, et al. Efeito da exposição à radiação ionizante de baixa dose na homeostase dinâmica de tiol-dissulfeto e níveis de albumina modificada por isquemia: estudo observacional [The effect of low dose ionizing radiation exposure on dynamic thiol-disulfide homeostasis and ischemia modified albumin levels: an observational study]. Braz J Anesthesiol 2020;70:233-9.

17. Brenner D, Elliston C, Hall E, Berdon W. Estimated risks of radiation-induced fatal cancer from pediatric CT. AJR Am J Roentgenol 2001;176:289-96.

18. Durović B, Spasić-Jokić V, Durović B. Influence of occupational exposure to low-dose ionizing radiation on the plasma activity of superoxide dismutase and glutathione level. Vojnosanit Pregl 2008;65:613-8.

19. Kłucinski P, Wójcik A, Grabowska-Bochenek R, et al. Erythrocyte antioxidant parameters in workers occupationally exposed to low levels of ionizing radiation. Ann Agric Environ Med 2008;15:9-12.

20. Shapira N, Scheuermann J, Perkins AE, et al. Quantitative positron emission tomography imaging in the presence of iodinated contrast media using electron density quantifications from dual-energy computed tomography. Med Phys 2021;48:273-86.

21. Song $H$, Guja KE, lagaru A. 18F-FDG PET/CT for Evaluation of Post-Transplant Lymphoproliferative Disorder (PTLD). Semin Nucl Med 2021;51:392-403.

22. Sher AC, Seghers V, Paldino MJ, et al. Assessment of Sequential PET/MRI in Comparison With PET/CT of Pediatric Lymphoma: A Prospective Study. AJR Am J Roentgenol 2016;206:623-31. 